Article

\title{
New Nonlocal Symmetries of Diffusion-Convection Equations and Their Connection with Generalized Hodograph Transformation
}

\author{
Valentyn Tychynin \\ Prydniprovs'ka State Academy of Civil Engineering and Architecture, 24a Chernyshevsky Street, \\ Dnipropetrovsk 49005, Ukraine; E-Mail: tychynin@ ukr.net
}

Academic Editor: Roman M. Cherniha

Received: 23 June 2015 / Accepted: 18 September 2015 / Published: 29 September 2015

\begin{abstract}
Additional nonlocal symmetries of diffusion-convection equations and the Burgers equation are obtained. It is shown that these equations are connected via a generalized hodograph transformation and appropriate nonlocal symmetries arise from additional Lie symmetries of intermediate equations. Two entirely different techniques are used to search nonlocal symmetry of a given equation: the first is based on usage of the characteristic equations generated by additional operators, another technique assumes the reconstruction of a parametrical Lie group transformation from such operator. Some of them are based on the nonlocal transformations that contain new independent variable determined by an auxiliary differential equation and allow the interpretation as a nonlocal transformation with additional variables. The formulae derived for construction of exact solutions are used.
\end{abstract}

Keywords: nonlocal symmetries; potential symmetries; formulae of nonlocal superposition; formulae for generation of solutions; generalized hodograph transformation

\section{Introduction}

We continue the nonlocal symmetries search of the diffusion-convection equations, connected by a generalized hodograph transformation (GHT) [1]. For this purpose we use the additional Lie symmetries (that are additional operators of invariance Lie algebras of higher dimension) of the intermediate equations arising at steps of this transformation, which consists of potential substitution and usual hodograph transformation. One can find many references and the extensive bibliography in researches devoted to studying the potential symmetries of nonlinear partial differential equations 
and systems [2-8]. The notion of potential symmetries of differential equations was introduced by Bluman et al. [2,3]. Later, Lisle proposed in $[9,10]$ the concept of potential equivalence transformations. It was successfully applied in [11] for deriving complete list of potential symmetries for wide classes of diffusion-convection equations.

On the other hand a number of interesting results for nonlinear equations are obtained for present day within the nonlocal transformations approach.

Analogously to continuous groups of usual transformations, the theory of groups of Lie-Bäcklund transformations, i.e., continuous groups of transformations involving derivatives of dependent variables, was developed by Anderson and Ibragimov [12]. An effective tool for this method application is the notion of recursion operator proposed by Olver [13].

A method for constructing nonlocally related PDE systems was introduced in [14,15]. Potential and nonlocal symmetries has been investigated for wide classes of nonlinear PDE systems there. It was shown that each point symmetry of a PDE system systematically yields a nonlocally related PDE system. Appropriate nonlocal symmetries were presented in the form including a potential variable.

The use of finite nonlocal transformations allowed to construct the formulae of generation of solutions and nonlocal nonlinear superposition for wide variety of nonlinear partial differential equations. This approach is based on the nonlocal transformations technic [16-21], which we regularly use for study of symmetries of the given nonlinear equations in [1] and in the current paper.

To start with, we remind the main concepts and notions which are necessary for understanding of the subsequent material. Let's assume that finite nonlocal transformation of variables

$$
\mathcal{T}: \quad x^{i}=h^{i}\left(y, v_{(k)}\right), \quad i=1, \ldots, n, \quad u^{K}=H^{K}\left(y, v_{(k)}\right), \quad K=1, \ldots, m
$$

exists and maps the given equation

$$
F_{1}\left(x, u_{(n)}\right)=0
$$

into the equation $\Phi\left(y, v_{(q)}\right)=0$ of order $q=n+k$. Suppose, that this equation admits a factorization to another equation

$$
F_{2}\left(y, v_{(s)}\right)=0
$$

i.e., $\Phi\left(y, v_{(q)}\right)=\lambda F_{2}\left(y, v_{(s)}\right)$. Here $\lambda$ is a differential operator of order $n+k-s$. Then we say that Equations (1) and (2) are connected by the nonlocal transformation $\mathcal{T}$

$$
F_{1}\left(x, u_{(n)}\right)=\lambda F_{2}\left(y, v_{(s)}\right)
$$

Here the symbol $u_{(r)}$ denotes the tuple of derivatives of the function $u$ from order zero up to order $r$. In the case of two independent variables we use the special notation of the variables: $x_{1}=x, x_{2}=t$ and thus $u_{t}=\partial u / \partial t=\partial_{t} u, u_{x}=\partial u / \partial x=\partial_{x} u$.

Nonlocal transformations can be effectively used for construction of the formulae generating solutions in both cases: for nonlocal invariance of equation

$$
F_{2}\left(y, v_{(n)}\right)=F_{1}\left(y, v_{(n)}\right)
$$

and when the connected equations are different. In a special case, when equation $F_{2}$ is linear, one can construct the formulae of nonlinear nonlocal superposition of solutions for nonlinear 
equation $F_{1}[1,17,22]$. We consider a case when the intermediate equations, connected by components of the generalized hodograph transformation exist, and these equations possess invariance Lie algebras of higher dimension. These, the last, we use for construction of corresponding nonlocal symmetries of the given equations and for deriving the formulae generating solutions.

The paper is organized as follows: In Section 2 we introduce a chain of the equations connected by steps of the GHT and compare the Lie invariance algebras obtained. Then (in Section 3) we construct nonlocal symmetries of these equations, which are generated by additional operators of invariance Lie algebras of the intermediate equations. Section 4 is devoted to the construction of finite nonlocal invariance transformations for a given equation and appropriate formulae generating their solutions. Namely, we combine the Lie symmetry transformation, generated by an additional operator $X$, which has been admitted by the intermediate equation, with a transformation, mapping this intermediate equation into the given one.

\section{Lie Symmetries of Given and Intermediate Equations}

We aim to consider two diffusion-convection equations from the class

$$
u_{t}-\partial_{x}\left(C(u)+K(u) \cdot u_{x}\right)=0
$$

which possess Lie invariance algebras of different dimensions. It is easy to check the mapping of the given equation

$$
u_{t}-\partial_{x}\left(\frac{1}{2} u^{-1}+u^{-2} u_{x}\right)=0
$$

into the Burgers equation

$$
z_{\eta}-\partial_{\xi}\left(-\frac{1}{2} z^{2}+z_{\xi}\right)=0
$$

via all steps of the GHT. This transformation was found first by Storm in [23] for linearization of nonlinear heat equations. Later this transformation was re-discovered in [24] and applied in [22,25] for investigation of various nonlinear heat equations. The GHT includes the potential substitution

$$
u=v_{x}(x, t)
$$

which is being applied to Equation (4) transforms it into the potential equation

$$
v_{t}-\frac{1}{2} v_{x}^{-1}-v_{x}^{-2} v_{x x}=0
$$

Then the hodograph transformation

$$
v(x, t)=\xi, \quad x=w(\xi, \eta), \quad t=\eta
$$

maps Equation (7) into the equivalent form

$$
w_{\eta}+\frac{1}{2} w_{\xi}^{2}-w_{\xi \xi}=0
$$

We will refer to Equation (9), Equation (7) obtained as "intermediate" equations. 
Having applied one more potential substitution

$$
w_{\xi}=z
$$

to Equations (9) we get the Burgers Equation (5). The composition of transformations Equations (6), (8) and (10) introduced above we mark further as $\mathcal{T}$.

Note that the relation of Equation (4) with the equation

$$
u_{t}-\partial_{x}\left(u^{-2} u_{x}\right)=0
$$

is a particular case of the more general result established in [26]. The results concerning linearization of Equation (11) were obtained by Rosen [27]. An example with Equation (11), particularly, was considered in $[14,15]$. Nonlocal symmetries were described using a potential variable. Here we apply a traditional approach [1] for searching nonlocal symmetries and aim to construct appropriate formulae of generation of solutions to equations considered. Then we use them for construction of exact solutions.

Consider some results of the classical Lie symmetry analysis for the equations connected by all components of the transformation $\mathcal{T}$. The maximal Lie algebra admitted by Equation (4) is four-dimensional (see for example [28])

$$
\begin{aligned}
& X_{1}=\partial_{t}, \quad X_{2}=\partial_{x}, \quad X_{3}=2 t \partial_{t}+u \partial_{u}, \\
& X_{4}=-2 \mathrm{e}^{\frac{1}{2} x} \partial_{x}+\mathrm{e}^{\frac{1}{2} x} u \partial_{u}
\end{aligned}
$$

while invariance algebra of the Burgers Equation (5) is spanned by five operators

$$
\begin{aligned}
& X_{1}=\partial_{\eta}, \quad X_{2}=\partial_{\xi}, \quad X_{3}=\eta \partial_{\xi}+\partial_{z}, \\
& X_{4}=\xi \partial_{\xi}+2 \eta \partial_{\eta}-z \partial_{z}, \\
& X_{5}=\eta \xi \partial_{\xi}+\eta^{2} \partial_{\eta}+(\xi-z \eta) \partial_{z}
\end{aligned}
$$

The operator $X_{5}$ does not correspond to Lie symmetry of Equation (4). The appropriate nonlocal symmetry of Equation (4) corresponding to the Lie symmetry $X_{5}$ of the Burgers equation has been investigated in details in [1]. Here we will take into account symmetries of the intermediate equations for construction of the appropriate nonlocal symmetries of two given Equations (4) and (5).

The maximal Lie invariance algebra of Equation (7) is spanned by the generators

$$
\begin{aligned}
& X_{1}=\partial_{t}, \quad X_{2}=\partial_{x}, \quad X_{3}=\partial_{v}, \quad X_{4}=v \partial_{x}+t \partial_{v} \\
& X_{5}=2 t \partial_{t}+v \partial_{v}, \quad X_{6}=\left(t+\frac{1}{2} v^{2}\right) \partial_{x}+t^{2} \partial_{t}+t v \partial_{v} \\
& X_{7}=b(v, t) \mathrm{e}^{\frac{1}{2} x} \partial_{x}, \quad b_{t}-b_{v v}=0
\end{aligned}
$$

Since Equation (9) is equivalent to Equation (7) it admits the Lie invariance algebra of the same structure

$$
\begin{aligned}
& X_{1}=\partial_{\eta}, \quad X_{2}=\partial_{\xi}, \quad X_{3}=\partial_{w}, \quad X_{4}=\eta \partial_{\xi}+\xi \partial_{w}, \\
& X_{5}=\xi \partial_{\xi}+2 \eta \partial_{\eta}, \quad X_{6}=\eta \xi \partial_{\xi}+\eta^{2} \partial_{\eta}+\left(\frac{1}{2} \xi^{2}+\eta\right) \partial_{w}, \\
& X_{7}=b(\xi, \eta) \mathrm{e}^{\frac{1}{2} w} \partial_{w}, \quad b_{\eta}-b_{\xi \xi}=0
\end{aligned}
$$

Note that both equations have two Lie symmetry generators more $\left(X_{3}\right.$ and $\left.X_{7}\right)$ than Equation (5) has. It will be interesting to compare the symmetries obtained above with those ones, which are admitted by a potential system, constructed for Equation (4)

$$
v_{x}-u=0
$$




$$
2 v_{t}-u^{-1}-2 u^{-2} u_{x}=0
$$

One can easily calculate the Lie symmetry of this system, which is spanned by generators

$$
\begin{aligned}
& X_{1}=\partial_{t}, \quad X_{2}=\partial_{x}, \quad X_{3}=\partial_{v}, \quad X_{4}=v \partial_{x}-u^{2} \partial_{u}+t \partial_{v} \\
& X_{5}=2 t \partial_{t}+u t \partial_{u}+v \partial_{v} \\
& X_{6}=\left(t+\frac{1}{2} v^{2}\right) \partial_{x}+t^{2} \partial_{t}+u(t-v u) \partial_{u}+t v \partial_{v} \\
& X_{7}=2 b(v, t) \mathrm{e}^{\frac{x}{2}} \partial_{x}-\left(2 u^{2} \partial_{v} b(v, t)+u\right) \mathrm{e}^{\frac{x}{2}} b(v, t) \partial_{u} \\
& b_{t}(v, t)-b_{v v}(v, t)=0
\end{aligned}
$$

Notice that to obtain an operator $X_{7}$ we chose it in the form

$$
X_{7}=2 b(v, t) \mathrm{e}^{\frac{x}{2}} \partial_{x}-\left(2 u^{2} a(x, t)-u\right) \mathrm{e}^{\frac{x}{2}} b(v, t) \partial_{u}
$$

where $a(x, t)$ and $b(v(x, t), t)$ are unknown differentiable functions.

Applying this operator to the system Equations (15) and (16), we get such conditions for these functions:

$$
\begin{gathered}
a(x, t) b(v(x, t), t)+D_{1} b(v(x, t), t)=0 \\
a(x, t) D_{1} b(v(x, t), t) u(x, t)+b(v(x, t), t) a_{x}(x, t)+D_{2} b(v(x, t), t) u(x, t)=0
\end{gathered}
$$

Here $D_{1} f(\alpha, \beta)$ means the derivative of a function $f(\alpha, \beta)$ with respect to its first argument. Substituting a solution of the first equation

$$
a(x, t)=-\frac{D_{1} b(v(x, t), t)}{b(v(x, t), t)}
$$

and an equality

$$
v_{x}=u
$$

into the second equation, we receive the equation for $b(v, t)$

$$
b_{t}(v, t)-b_{v v}(v, t)=0
$$

In what follows we will use two entirely different techniques to search for nonlocal symmetry of a given equation. The first is based on usage of the characteristic equation generated by an additional operator. Another technique assumes reconstruction of a parametrical Lie group transformation from such operator.

\section{Nonlocal Symmetries Generated by Additional Lie Symmetries of the Intermediate Equations}

Here we apply additional operators of Lie algebras obtained for intermediate equations to search appropriate nonlocal symmetry of given equations and construct the formulae of generation their solutions. 


\subsection{The Operator X 7th Case}

Comparison of Lie algebras Equations (12) and (14) shows that Equation (9) admits an additional (with respect to Equation (5)) generator $X_{7}$. The characteristic equation corresponding to sum $X_{7}+X_{3}$ of generators of algebra Equation (14) has the form

$$
w+2 \ln |b|=0
$$

One can easily identify this formula with the known substitution linearizing Equation (9). To present this expression via the variable $z(\xi, \eta)$ we will differentiate it with respect to $\xi$

$$
w_{\xi}+2 b_{\xi} b^{-1}=0
$$

Then, substituting $w_{\xi}=z$ into above equation, we find the characteristic equation which determines appropriate nonlocal symmetry of Equation (5)

$$
z+2 b_{\xi} b^{-1}=0
$$

Therefore the "lacking" operator for the Burgers equation should be written in the form

$$
X_{7}^{\star}=\left(z+2 \partial_{\xi} \ln b\right) \partial_{z}
$$

Note that an expression Equation (23) is well known Cole-Hopf substitution.

Conversely, applying the transformation Equation (8) with its differential consequence $w_{\xi}=1 / v_{x}$ to the Equation (22), we find nonlocal symmetry for Equation (7)

$$
v_{x}+b / 2 b_{v}=0
$$

Taking into account substitution Equation (20), this equation takes the form

$$
u+b / 2 b_{v}=0
$$

Here $b(v, t)$ is an arbitrary solution of the linear heat Equation (21).

An application of the nonlocal transformation Equation (25) to Equation (4) generates an expression which after simplification vanishes identically on the manifold defined by Equation (7) and expression $v_{x}+b / 2 b_{v}=0$ with its differential consequences.

Usage of a classical linear superposition principle in Equation (25) allows one to get the formula generating solutions of Equation (4)

$$
u^{\mathrm{I}}+b^{\mathrm{I}}\left(2 b_{v}^{\mathrm{I}}\right)^{-1}=0, u^{\mathrm{II}}+b^{\mathrm{II}}\left(2 b_{v}^{\mathrm{II}}\right)^{-1}=0, \quad \rightarrow \quad u^{\mathrm{III}}=-\left(b^{\mathrm{I}}+b^{\mathrm{II}}\right)\left(2 b_{v}^{\mathrm{I}}+2 b_{v}^{\mathrm{II}}\right)^{-1}
$$

On the other hand the generator $X_{7}$ of the Lie invariance algebra of the system Equations (15) and (16) determines a potential symmetry of Equation (4) and admits presentation by the characteristic equation

$$
u_{x}+u^{2} \frac{b_{v}(v, t)}{b(v, t)}+\frac{1}{2} u=0
$$

where $u(x ; t)$ and $b(v ; t)$ satisfy Equations (21) and (20). Thus, the appropriate "lacking" operator for Equation (4) should be written in the form, depending on $b(v, t)$

$$
X_{7}^{\star}=b(v, t) \mathrm{e}^{\frac{1}{2} x} \partial_{x}-\left(u^{2} \frac{b_{v}(v, t)}{b(v, t)}+\frac{1}{2} u\right) b(v, t) \mathrm{e}^{\frac{1}{2} x} \partial_{u}
$$


Theorem 1. The characteristic Equation (27) determines nonlocal transformation with additional variables, which connects Equations (4) and (7) taking into account Equation (21) and condition Equation (20). Additional independent variable $v(x, t)$ is determined by Equation (7) and additional dependent variable $b(v, t)$ is an arbitrary solution of the Equation (21).

To prove the statement formulated above we will first rewrite Equation (27), using equality Equation (20) in the form

$$
\partial_{x} \ln |u|+\partial_{x} \ln |b(v, t)|+\frac{1}{2}=0
$$

and then integrate the result with respect to $x$

$$
u(x, t)=\frac{s(t) \mathrm{e}^{-1 / 2 x}}{b(v, t)}
$$

Having applied this transformation to Equation (4) and use the conditions

$$
b_{t}(v, t)-b_{v v}(v, t)=0, \quad v_{x x}=v_{t} v_{x}^{2}-1 / 2 v_{x}, \quad v_{x}(x, t)=\frac{s(t) \mathrm{e}^{-1 / 2 x}}{b(v, t)}
$$

we obtain a result, which vanishes identically after simplification.

The above assertion admits another form.

Theorem 2. The Equation (4) is invariant under action of the prolonged operator $X_{7}^{\star}$ on the manifold, determined by Equation (7), condition Equation (20) and the Equation (21) with their differential consequences.

To deduce the characteristic equation, which corresponds to Equation (29) and determines nonlocal symmetry of the Equation (4) in terms of variables $x, t, u(x, t)$, we need to exclude a variable $v(x, t)$ from expression Equation (30), where $b(v, t)$ is a function belonging to the set of solutions of the linear heat Equation (21).

New designations will be necessary for us in what follows. Suppose that we can solve the functional equation $b(v, t)=a$ with respect to the first argument of $b$. Then a function $\beta(a, t): b(v, t)=a$ $\rightarrow \quad v=\beta(a, t)$, will denote a solution of this equation. Therefore

$$
v(x, t)=\beta\left(\frac{s(t) \mathrm{e}^{-1 / 2 x}}{u}, t\right)
$$

is a solution of Equation (30) with respect to $v$. Using potential substitution Equation (20) we obtain equality

$$
u(x, t)-\partial_{x} \beta\left(\frac{s(t) \mathrm{e}^{-1 / 2 x}}{u(x, t)}, t\right)=0
$$

Differentiating and simplifying this expression, we get the presentation of the onetime integrated characteristic equation which determines nonlocal symmetry of Equation (4)

$$
\begin{aligned}
& 2 u^{3}+D_{1} \beta\left(\frac{s(t) \mathrm{e}^{-1 / 2 x}}{u}, t\right) s(t) \mathrm{e}^{-1 / 2 x} u \\
& +2 D_{1} \beta\left(\frac{s(t) \mathrm{e}^{-1 / 2 x}}{u}, t\right) s(t) \mathrm{e}^{-1 / 2 x} u_{x}=0
\end{aligned}
$$

$D_{1} f(\alpha, \beta)$ means the derivative of a function $f(\alpha, \beta)$ with respect to its first argument. 
For instance, a given simple solution of the linear heat Equation (21)

$$
b(v, t)=\left(c_{1} \mathrm{e}^{\sqrt{\alpha} v}+c_{2} \mathrm{e}^{-\sqrt{\alpha} v}\right) c_{3} \mathrm{e}^{\alpha t}
$$

allows one to obtain the expression Equation (30) in such particular form:

$$
\left(c_{1} \mathrm{e}^{\sqrt{\alpha} v}+c_{2} \mathrm{e}^{-\sqrt{\alpha} v}\right) c_{3} \mathrm{e}^{\alpha t}-\frac{s(t) \mathrm{e}^{-1 / 2 x}}{u(x, t)}=0
$$

Here $\alpha, c_{i},(i=1,2,3)$ are arbitrary constants. This equation has two solutions for $v(x, t)$

$$
v=\ln \left|\frac{s(t) \mathrm{e}^{-1 / 2 x} \pm \sqrt{s^{2}(t) \mathrm{e}^{-x}-4 c_{3}^{2} \mathrm{e}^{2 \alpha t} u^{2} c_{1}} c_{2}}{2 c_{1} c_{3} \mathrm{e}^{\alpha t} u}\right|(\sqrt{\alpha})^{-1}
$$

Excluding the variable $v$ from Equation (31), we can rewrite it as follows

$$
u-\partial_{x}\left(\ln \left|\frac{s(t) \mathrm{e}^{-1 / 2 x} \pm \sqrt{s^{2}(t) \mathrm{e}^{-x}-4 c_{3}^{2} \mathrm{e}^{2 \alpha t} u^{2} c_{1} c_{2}}}{2 c_{1} c_{3} \mathrm{e}^{\alpha t} u}\right|(\sqrt{\alpha})^{-1}\right)=0
$$

Finally we obtain two the onetime integrated characteristic equations which determine nonlocal symmetries of Equation (4)

$$
2 u_{x}+2 \sqrt{\alpha} u^{2}+u \pm \frac{8 \sqrt{\alpha} c_{1} c_{2} c_{3}^{2} \mathrm{e}^{2 \alpha t} u^{4}}{\sqrt{s^{2}(t) \mathrm{e}^{-x}-4 c_{3}^{2} \mathrm{e}^{2 \alpha t} u^{2} c_{1} c_{2}}+s(t) \mathrm{e}^{-1 / 2 x}}=0
$$

Choosing in Equation (32), in particular, $c_{2}=0, c_{3}=1$, and integrating this equation, we obtain two ansatzes

$$
u= \pm \frac{1}{2 \sqrt{\alpha}-g(t) \mathrm{e}^{1 / 2 x}}
$$

which contain arbitrary functions of $t$, which must be specialized by Equation (4). So, finally we obtain two solutions of this equation

$$
u= \pm \frac{1}{2 \sqrt{\alpha}-k \mathrm{e}^{1 / 2 x}}
$$

Note that such functions can be obtained by means of the Lie ansatz.

Theorem 3. The operator Equation (28) allows one to construct the associated transformation determined by the formulas

$$
\begin{gathered}
2\left(\mathrm{e}^{\frac{x^{\mathrm{I}}}{2}}+\mathrm{e}^{\frac{x^{\mathrm{II}}}{2}}\right)+\varepsilon b^{\mathrm{I}}\left(v^{\mathrm{I}}\left(x^{\mathrm{I}}, t\right), t\right) \mathrm{e}^{\frac{x^{\mathrm{I}}+x^{\mathrm{II}}}{2}}=0 \\
u^{\mathrm{II}}\left(x^{\mathrm{II}}, t\right)=-\frac{u^{\mathrm{I}}\left(x^{\mathrm{I}}, t\right)\left(-2+b^{\mathrm{I}}\left(v^{\mathrm{I}}\left(x^{\mathrm{I}}, t\right), t\right) \varepsilon \mathrm{e}^{\frac{x^{\mathrm{I}}}{2}}\right)}{2+2 u^{\mathrm{I}}\left(x^{\mathrm{I}}, t\right) D_{1} b^{\mathrm{I}}\left(v^{\mathrm{I}}\left(x^{\mathrm{I}}, t\right), t\right) \varepsilon \mathrm{e}^{\frac{x^{\mathrm{I}}}{2}}}
\end{gathered}
$$

where $\partial_{x^{\mathrm{I}}} v^{\mathrm{I}}\left(x^{\mathrm{I}}, t\right)=u^{\mathrm{I}}\left(x^{\mathrm{I}}, t\right)$ and $b^{\mathrm{I}}\left(v^{\mathrm{I}}\left(x^{\mathrm{I}}, t\right), t\right)$ is any arbitrary solution of the Equation (21).

To prove this theorem we restore a Lie group transformation using the operator Equation (28). So, we obtain

$$
\begin{aligned}
& 2\left(\mathrm{e}^{\frac{x}{2}}+\mathrm{e}^{\frac{r}{2}}\right)+\varepsilon b(v(x, t), t) \mathrm{e}^{\frac{x+r}{2}}=0, \quad s=t, \\
& p(r, s)=-\frac{u(x, t)\left(-2+b(v(x, t), t) \varepsilon \mathrm{e}^{\frac{x}{2}}\right)}{2+2 u(x, t) D_{1} b(v(x, t), t) \varepsilon \mathrm{e}^{\frac{x}{2}}}
\end{aligned}
$$


Making here change of notations for new variables, we find the Equations (33) and (34).

The algorithm of generation of solutions for Equation (4) based on these formulae works, as follows. Let $u^{\mathrm{I}}\left(x^{\mathrm{I}}, t\right)$ be any known solution of Equation (4), then $v^{\mathrm{I}}\left(x^{\mathrm{I}}, t\right)=\int u^{\mathrm{I}}\left(x^{\mathrm{I}}, t\right) d x^{\mathrm{I}}+s(t)$ can be found. Here $s(t)$ is arbitrary function which can be specialized by Equation (29). Substituting obtained $v^{\mathrm{I}}$ and arbitrary solution of the linear heat equation $b^{\mathrm{I}}\left(v^{\mathrm{I}}\left(x^{\mathrm{I}}, t\right), t\right)$ into Equation (33) and solving the result with respect to $x^{\mathrm{I}}$, we get $x^{\mathrm{I}}=\chi\left(x^{\mathrm{I}}, t\right)$. Finally, inserting this $x^{\mathrm{I}}$ into Equation (34) we find a new solution of Equation (4).

Example 1. Choosing $u^{\mathrm{I}}=\mathrm{e}^{-\frac{x^{\mathrm{I}}}{2}}$ and integrating it with respect to $x$, we obtain $v^{\mathrm{I}}=-2 \mathrm{e}^{-\frac{x^{\mathrm{I}}}{2}}+s(t)$. Specializing arbitrary function by Equation (29), we get $v^{\mathrm{I}}=-2 \mathrm{e}^{-\frac{x^{\mathrm{I}}}{2}}+c_{1}$. Set arbitrary solution of the linear heat equation $b^{\mathrm{I}}=\mathrm{e}^{c_{2} t+\sqrt{c_{2}} v^{\mathrm{I}}}$, then $D_{1} b^{\mathrm{I}}=\sqrt{c_{2}} \mathrm{e}^{c_{2} t+\sqrt{c_{2}} v^{\mathrm{I}}}$. Rewriting these formulae and taking into account $v^{\mathrm{I}}=-2 \mathrm{e}^{\frac{x^{\mathrm{I}}}{2}}+c_{1}$, we find

$$
b^{\mathrm{I}}=\mathrm{e}^{c_{2} t+\sqrt{c_{2}}\left(-2 \mathrm{e}^{\frac{x^{\mathrm{I}}}{2}}+c_{1}\right)}
$$

and

$$
D_{1} b^{\mathrm{I}}=\sqrt{c_{2}} \mathrm{e}^{c_{2} t+\sqrt{c_{2}}\left(-2 \mathrm{e}^{\frac{x^{\mathrm{I}}}{2}}+c_{1}\right)}
$$

Substituting a found above $b^{\mathrm{I}}$ into Equation (33) and solving this result with respect to $x^{\mathrm{I}}$, we get $x^{\mathrm{I}}=\chi\left(x^{\mathrm{II}}, t\right)$

$$
x^{\mathrm{I}}=x^{\mathrm{II}}-2 \ln \left|\frac{1}{2} \operatorname{LambertW}\left(\sqrt{c_{2}} \varepsilon \mathrm{e}^{\left(c_{1} \sqrt{c_{2}}+t c_{2}-2 \sqrt{c_{2}} \mathrm{e}^{-\frac{x^{\mathrm{II}}}{2}}\right) \mathrm{e}^{\frac{x^{\mathrm{II}}}{2}}}+\sqrt{c_{2}}\right)\right|+\ln \left|c_{2}\right|
$$

After simplification Equation (34) with obtained $x^{\mathrm{I}}=\chi\left(x^{\mathrm{II}}, t\right), b^{\mathrm{I}}$ and $D_{1} b^{\mathrm{I}}$ we find a new solution of Equation (4)

$$
\begin{gathered}
u^{\mathrm{II}}\left(x^{\mathrm{II}}, t\right)=\mathrm{e}^{c_{1} \sqrt{c_{2}}+t c_{2}-2 \sqrt{c_{2}} \mathrm{e}^{-\frac{x^{\mathrm{II}}}{2}}-\frac{x^{\mathrm{II}}}{2}} G^{-1} \\
G=\mathrm{e}^{c_{1} \sqrt{c_{2}}+t c_{2}-2 \sqrt{c_{2}}} \mathrm{e}^{-\frac{x^{\mathrm{II}}}{2}}\left(\operatorname{LambertW}\left(\sqrt{c_{2}} \varepsilon \mathrm{e}^{\left(c_{1} \sqrt{c_{2}}+t c_{2}-2 \sqrt{c_{2}} \mathrm{e}^{-\frac{x^{\mathrm{II}}}{2}}\right)}\right)+1\right)
\end{gathered}
$$

\subsection{The Operator X 4th Case}

The Lie invariance algebra Equation (13) of Equation (7) includes one operator $X_{4}=v \cdot \partial_{x}+t \cdot \partial_{v}$ more, than invariance algebra of Equation (4) has. This operator yields the characteristic equation

$$
v \cdot v_{x}-t=0
$$

Differentiating Equation (35) with respect to $x$ and using substitution Equation (20) we obtain

$$
v u_{x}+u^{2}=0
$$

Excluding $v(x, t)$ from this equation we get the second order differential equation for $u$ which determines nonlocal symmetry of Equation (4)

$$
-3 u_{x}^{2}+u u_{x x}=0
$$


Solving Equation (37), we find non-Lie ansatzes of Equation (4)

$$
u= \pm\left(\sqrt{-2 F_{1}(t) x-2 F_{2}(t)}\right)^{-1}
$$

Substitution them into Equation (4) and splitting a result with respect to $x$ yields the reduced system of two ordinary differential equations

$$
\dot{F}_{1}(t)-\left(F_{1}(t)\right)^{2}=0, \quad \dot{F}_{2}(t)-F_{1}(t) F_{2}(t)-\left(F_{1}(t)\right)^{2}=0
$$

Solution of this system

$$
F_{1}(t)=-\left(t-c_{2}\right)^{-1}, \quad F_{2}(t)=\frac{\ln \left|t-c_{2}\right|-c_{1}}{t-c_{2}}
$$

allows us to construct appropriate solutions of Equation (4)

$$
u= \pm \frac{\sqrt{2}}{2} \sqrt{\frac{t-c_{2}}{x-\ln \left|t-c_{2}\right|+c_{1}}}
$$

The result obtained is closely connected with potential symmetry of Equation (4) determined by the generator $X_{4}$ of Lie algebra Equation (17). The characteristic equations corresponding to this operator, are

$$
\begin{gathered}
v \cdot u_{x}+u^{2}=0 \\
v \cdot v_{x}-t=0
\end{gathered}
$$

They, obviously, coincide with Equations (36) and (35) accordingly. The solution of this system

$$
u=\frac{t}{\sqrt{2 \cdot x \cdot t+F_{1}(t)}+F_{2}(t) \cdot t}, \quad v=\sqrt{2 x t+F_{1}(t)}
$$

yields the reduced system of ordinary differential equations

$$
F_{2}(t)=0, \quad t \cdot \dot{F}_{1}-F_{1}(t)+2 t=0
$$

and, consequently, yields appropriate solution to the system Equations (39) and (40)

$$
u=\frac{\sqrt{t}}{\sqrt{2 x-2 \ln |t|+c_{1}}}, \quad v=\sqrt{t} \sqrt{2 x-2 \ln |t|+c_{1}}
$$

If we set $c_{2}=0$ in Equation (38), we will obtain the previous expression for the function $u$.

\section{Nonlocal Invariance Transformations and Generation of Solutions}

Here we construct the one-parameter group of Lie symmetries generated by operator, admitted by the potential system Equations (15) and (16), and it is used for construction of the corresponding formula for generation of solutions of Equation (4). This involves a successive implementation of the transformations $\mathcal{T}$ and the one-parameter group of point transformations associated with the corresponding infinitesimal operator which is admitted by the potential system. 
Assume that the partial differential equation

$$
F\left(x, t, u_{(k)}\right)=0
$$

should admit at least one conservation law

$$
D_{t} \phi^{t}\left(x, t, u_{(r)}\right)+D_{x} \phi^{x}\left(x, t, u_{(r)}\right)=0
$$

where $D_{t}$ and $D_{x}$ are total derivatives with respect to the variables $t$ and $x, \phi^{t}$ and $\phi^{x}$ are conserved density and flux, respectively. When Equation (41) admits representation Equation (42) there exists the potential function $v$ determined by the auxiliary system [2]

$$
v_{x}=\phi^{t}\left(x, t, u_{(r)}\right), \quad v_{t}=-\phi^{x}\left(x, t, u_{(r)}\right)
$$

Potential symmetry of the Equation (41) is determined by the Lie symmetry generator

$$
X=\xi_{1}(x, t, u, v) \partial_{x}+\xi_{2}(x, t, u, v) \partial_{t}+\eta_{1}(x, t, u, v) \partial_{u}+\eta_{2}(x, t, u, v) \partial_{v}
$$

admitted by system Equation (43) with at least one nonzero partial derivative

$$
\partial_{v} \xi_{1}(x, t, u, v), \quad \partial_{v} \xi_{1}(x, t, u, v), \quad \partial_{v} \eta_{1}(x, t, u, v)
$$

In this case the one-parameter Lie point symmetry group associated with infinitesimal generator $X$ exists

$$
\begin{aligned}
& r=x^{\prime}=f_{1}(x, t, u, v ; \varepsilon), \quad s=t^{\prime}=f_{2}(x, t, u, v ; \varepsilon), \\
& p(r, s)=u^{\prime}=g_{1}(x, t, u, v ; \varepsilon), \quad q(r, s)=v^{\prime}=g_{2}(x, t, u, v ; \varepsilon)
\end{aligned}
$$

Here $\varepsilon$ is a group parameter.

As Equation (4) is connected with the potential system Equations (15) and (16), one can construct the projection of the corresponding transformation Equation (44) onto the space of variables $(x, t, u(x, t))$ using substitution Equation (20)

$$
\begin{aligned}
& r=x^{\prime}=f_{1}\left(x, t, u, \int u(x, t) \mathrm{d} x ; \varepsilon\right), \\
& s=t^{\prime}=f_{2}\left(x, t, u, \int u(x, t) \mathrm{d} x ; \varepsilon\right), \\
& p(r, s)=u^{\prime}=g_{1}\left(x, t, u, \int u(x, t) \mathrm{d} x ; \varepsilon\right)
\end{aligned}
$$

This transformation is the finite Lie-Bäcklund transformation which leaves Equation (4) nonlocal-invariant. Stated above allows formulating the following statement.

Theorem 4. Equation (4) is nonlocal-invariant under the transformations

$$
\begin{gathered}
r=\ln \left|\frac{2}{2-\varepsilon t}\right|+\frac{\left(\int u \mathrm{~d} x\right)^{2} \varepsilon}{4-2 \varepsilon t}+x, \\
s=\frac{2 t}{2-\varepsilon t}, \quad p(r, s)=\frac{-2 u}{\varepsilon t-u \varepsilon \int u \mathrm{~d} x-2} ; \\
r=2 \ln \left|-2\left(-2+b\left(\int u \mathrm{~d} x, t\right) \varepsilon \mathrm{e}^{\frac{1}{2} x}\right)^{-1}\right|+x, \quad s=t, \\
p(r, s)=-\frac{u\left(-2+b\left(\int u \mathrm{~d} x, t\right) \varepsilon \mathrm{e}^{\frac{1}{2} x}\right)}{2+2 u D_{1}(b)\left(\int u \mathrm{~d} x, t\right) \varepsilon \mathrm{e}^{\frac{1}{2} x}}
\end{gathered}
$$

and

$$
r=\frac{1}{2} \varepsilon^{2} t+\varepsilon \int u \mathrm{~d} x+x, s=t, p(r, s)=\frac{u}{u \varepsilon+1}
$$


The proof of this statement for transformation Equation (46). Let us choose, for instance, the operator

$$
X_{6}=\left(t+\frac{1}{2} v^{2}\right) \partial_{x}+t^{2} \partial_{t}+(-v u+t) u \partial_{u}+t v \partial_{v}
$$

This operator belongs to the Lie invariance algebra Equation (17) of the system Equations (15) and (16). The corresponding Lie group transformation has the form

$$
\begin{gathered}
r=\ln \left|\frac{2}{2-\varepsilon t}\right|+\frac{v^{2} \varepsilon}{4-2 \varepsilon t}+x, s=\frac{2 t}{2-\varepsilon t}, \\
p(r, s)=-\frac{2 u}{\varepsilon t-v u \varepsilon-2}, q(r, s)=\frac{2 v}{2-\varepsilon t}
\end{gathered}
$$

Excluding in these formulae $v$ with the help of expression $v=\int u(x, t) \mathrm{d} x$, we get a nonlocal transformation in space of variables $r, s, p$ Equation (46). Then we apply the transformation Equation (46) to Equation (4), rewritten in new designations

$$
\partial_{s} p(r, s)-\partial_{r}\left(\frac{1}{2} p(r, s)^{-1}+p(r, s)^{-2} \partial_{r} p(r, s)\right)=0
$$

and substitute the integro-differential consequences of Equation (4)

$$
u_{x x}=u^{2} u_{t}+\frac{1}{2} u_{x}+2 u^{-1} u_{x}^{2}
$$

and

$$
\int u_{t} \mathrm{~d} x=\frac{1}{2} u^{-1}+u^{-2} u_{x}
$$

into the obtained result. After simplification the last vanishes identically.

To prove this statement for transformation Equation (47) we consider the generator of Lie algebra Equation (17)

$$
X_{7}=\mathrm{e}^{\frac{1}{2} x} b(v, t) \partial_{x}-u\left(\frac{u b_{v}}{b(v, t)}+\frac{1}{2}\right) b(v, t) \mathrm{e}^{\frac{1}{2} x} \partial_{u}
$$

where $D_{2} b(v, t)-D_{11} b(v, t)=0, v=v(x, t)$, which yields the group-invariant transformation of the potential system Equations (15) and (16)

$$
\begin{gathered}
r=2 \ln \left|-2\left(-2+b(v, t) \varepsilon \mathrm{e}^{1 / 2 x}\right)^{-1}\right|+x, \quad s=t, \\
p(r, s)=-\frac{u\left(-2+b(v, t) \varepsilon \mathrm{e}^{1 / 2 x}\right)}{2+2 u D_{1}(b)(v, t) \varepsilon \mathrm{e}^{1 / 2 x}}, \quad q(r, s)=v
\end{gathered}
$$

Hereinafter $D_{i}(f)(\alpha, \beta)$ and $D_{i, j}(f)(\alpha, \beta),(i, j=1,2)$ denote total derivatives of a function $f(\alpha, \beta)$ with respect to $i$-th and $j$-th variables of the first and second order accordingly, $\varepsilon$ is a group parameter associated with the operator $X_{7}$. Having substituted into previous formulae $v(x, t)=\int u \mathrm{~d} x$ we get a nonlocal transformation Equation (47).

To verify invariance of Equation (50) we apply to it Equation (47) and substitute Equations (51) and (52) and expressions

$$
\begin{gathered}
D_{11} b\left(\int u \mathrm{~d} x, t\right)=D_{2} b\left(\int u \mathrm{~d} x, t\right) \\
D_{111} b\left(\int u \mathrm{~d} x, t\right)=D_{12} b\left(\int u \mathrm{~d} x, t\right) \\
D_{11} u(x, t)=\frac{2 D_{2} u(x, t)(u(x, t))^{3}+u(x, t) D_{1} u(x, t)+4\left(D_{1} u(x, t)\right)^{2}}{2 u(x, t)}
\end{gathered}
$$


into the obtained result. The expression obtained vanishes identically after simplification.

The proof of Theorem 4 for transformation Equation (48). Another operator of the Lie invariance algebra Equation (17)

$$
X_{4}=v \partial_{x}-u^{2} \partial_{u}+t \partial_{v}
$$

generates the finite Lie-group transformation

$$
r=\frac{1}{2} \varepsilon^{2} t+\varepsilon v+x, \quad s=t, \quad p(r, s)=\frac{u}{u \varepsilon+1}, q(r, s)=v+\varepsilon t
$$

Projection of this transformation onto the space of variables $(x, t, u(x, t))$ admits the form Equation (48). This transformation leaves the Equation (4) nonlocal-invariant. The statement can be proved like in the previous cases.

Let us consider some applications of the Theorem 4. If $p(r, s)$ is a known solution of the Equation (50), then the new solution of Equation (4) can be constructed by means of solution Equation (46) after subsequent specialization of the arbitrary function appearing as a result of integration in it.

Example 2. Inserting the solution of Equation (4) $u(x, t)=-\frac{1}{2} \mathrm{e}^{-\frac{1}{2} x}$ into Equation (46), we obtain the formulae of transformation, which contain an arbitrary function $F(t)$

$$
\begin{aligned}
& r=\ln \left|\frac{2}{2-t \varepsilon}\right|+x+\frac{\varepsilon\left(\mathrm{e}^{-\frac{1}{2} x}+F(t)\right)^{2}}{4-2 t \varepsilon}, \\
& s=\frac{-2 t}{-2+\varepsilon t}, \quad p(r, s)=\frac{2\left(-\frac{1}{2} \mathrm{e}^{-\frac{1}{2} x}\right)}{2 \varepsilon t-\frac{1}{2} \mathrm{e}^{-\frac{1}{2} x}\left(\mathrm{e}^{-\frac{1}{2} x}+F(t)\right) \varepsilon-4}
\end{aligned}
$$

Specializing $F(t)$ by Equation (4) and supposing $F(t)=0$ for simplicity of evaluations, we obtain

$$
\begin{aligned}
& r=\ln \left|\frac{2}{2-t \varepsilon}\right|+x+\frac{\varepsilon \mathrm{e}^{-x}}{4-2 t \varepsilon}, \\
& s=\frac{-2 t}{-2+\varepsilon t}, \quad p(r, s)=\frac{2 \mathrm{e}^{-1 / 2 x}}{2 \varepsilon t+\mathrm{e}^{-x} \varepsilon-4}
\end{aligned}
$$

Solving two first equations of the Equation (55) with respect to $x$ and $t$, we get

$$
x=r+\text { LambertW }\left(-\frac{1}{16} \frac{\varepsilon(\varepsilon s+2)^{2}}{\mathrm{e}^{r}}\right)+\ln \left|2(\varepsilon s+2)^{-1}\right|, \quad t=\frac{2 s}{\varepsilon s+2}
$$

Substituting these $x, t$ into the third equation of system Equation (55) we receive after simplification the solution of Equation (50)

$$
\begin{gathered}
p(r, s)=-\frac{\sqrt{\varepsilon s+2}}{\sqrt{2}} \mathrm{e}^{-1 / 2 r}\left(1+\text { LambertW }\left(-\frac{1}{16} \varepsilon(\varepsilon s+2)^{2} \mathrm{e}^{-r}\right)\right)^{-1} \\
\sqrt{-\varepsilon^{-1} \mathrm{e}^{r} \text { LambertW }\left(-\frac{1}{16} \varepsilon(\varepsilon s+2)^{2} \mathrm{e}^{-r}\right)}
\end{gathered}
$$

Example 3. Let us apply Equation (46) to the solution $u(x, t)=\frac{t}{\sqrt{2 t(x-\ln |t|)}}$. We choose for simplicity the arbitrary function appearing as a result of integration, equal to zero. The change of the independent variables has such a form:

$$
\begin{aligned}
& x=\ln \left|\frac{2 s}{\varepsilon s+2}\right|+\frac{2 r-2 \ln |s|}{\varepsilon s+2}, \\
& t=2 \frac{s}{\varepsilon s+2}
\end{aligned}
$$

After substitution of new independent variables into the expression

$$
p(x, t)=\sqrt{\frac{t}{2(x-\ln |t|)}}
$$


we finally get the same solution of the Equation (4)

$$
p(r, s)=\sqrt{\frac{s}{2(r-\ln |s|)}}
$$

Hence, this solution is an invariant solution with respect to the nonlocal transformation considered.

Equations (47) and (48) too allow generating new solutions of the Equation (4) if $p(r ; s)$ is its known solution.

The operator $X_{4}$ generates two corresponding characteristic equations

$$
\begin{gathered}
v v_{x}-t=0, \\
v u_{x}+u^{2}=0
\end{gathered}
$$

The first equation determines usual Lie point symmetry of Equation (7). Equation (57) determines potential symmetry of Equation (4). Both these equations are connected by the potential substitution Equation (20).

Solving Equation (57) with respect to $v$

$$
v=-u^{2} u_{x}^{-1}
$$

and substituting this into Equation (56), we obtain the second order differential equation, determining nonlocal symmetry of Equation (4)

$$
u_{x x} u^{4}-2 u_{x}^{2} u^{3}+t u_{x}^{3}=0
$$

and, consequently, appropriate nonlocal ansatzes for a given equation

$$
u=\frac{-f_{1}(t) \pm \sqrt{f_{1}(t)^{2}+2 t f_{2}(t)+2 t x}}{2\left(f_{2}(t)+x\right)}
$$

Here $f_{i},(i=1,2,3)$ are the arbitrary functions of variable $t$. This ansatz can be used for construction of solutions to Equation (4).

Let us describe symmetry of Equation (4) corresponding to Lie symmetry Equation (56) of Equation (7). First we substitute $v_{x}=u$ into Equation (56)

$$
v v_{x}-t=v u-t=0
$$

and solve it for $v(x, t)$. Differentiating the result with respect to $x$, we get

$$
v_{x}+t \cdot u^{-2} u_{x}=0
$$

Note that if we wrote obtained above expression using Equation (20) in a form $u^{3}+t u_{x}=0$, we would make a mistake, because such symmetry is not admitted by Equation (4).

So, using substitution $v v_{x}-t=0$ in the previous expression, we get

$$
u^{2}+v u_{x}=0
$$


(the second characteristic equation for $X_{4}$ ). Solving the last equation for $v(x, t)$ and differentiating it with respect to $x$, we obtain

$$
v_{x}=-2 u+u^{2} u_{x}^{-2} \cdot u_{x x}
$$

Using here potential substitution Equation (20) and simplifying result, we get corresponding nonlocal symmetry of the Equation (4)

$$
u_{x x} u-3 u_{x}^{2}=0
$$

Integrating this equation we find two ansatzes for Equation (4)

$$
u= \pm \frac{1}{\sqrt{-2 f_{1}(t) x-2 f_{2}(t)}}
$$

They depend on arbitrary functions $f_{1}(t)$ and $f_{2}(t)$. Specializing them by Equation (4), we find two solutions

$$
u= \pm \frac{\sqrt{t-c_{2}}}{\sqrt{2 c_{1} \mathrm{e}^{3}-2 \ln \left|-t+c_{2}\right|+2\left(x+c_{3}\right)}}
$$

Since Equation (4) is invariant with respect to the time and space translations generated by the operators $X_{1}$ and $X_{2}$ the last solution, Equation (38) and $u$-part of solution to the system Equations (39) and (40) represent the same exact solution.

\section{Conclusions}

Two given diffusion-convection equations, which allow connection by the generalized hodograph transformation are considered. It is shown in this paper that the intermediate equations possess the additional (with respect to given equations) operators of invariance Lie algebras. The appropriate additional operators allow to construct potential and nonlocal symmetries of the given differential equations. We have constructed the one-parameter groups of Lie symmetries generated by operators, admitted by the potential system Equations (15) and (16), and use them for construction of the corresponding formulae for generation of solutions of Equation (4). This involves a successive implementation of appropriate transformations $\mathcal{T}$ and the one-parameter group of point transformations associated with the corresponding infinitesimal operator admitted by the potential system. New formulae for generation of solutions have been constructed. Some of them are based on the nonlocal transformations that contain new independent variable determined by an auxiliary differential equation. This type of transformations allows interpretation as an example of the nonlocal transformation with additional variables. The obtained formulae allow transformation of a known solution of the given equation into another its solution. They have been used for construction of exact solutions, some of which are new. They are either obtained in explicit form or presented in a new parametrical representations, where a functional parameter is given implicitly. All found solutions can be extended to parametric families by means of the Lie symmetry transformations or by using other formulae for generation of solutions.

\section{Acknowledgments}

The author thanks the referees for a lot of helpful remarks and suggestions. 


\section{Conflicts of Interest}

The author declares no conflict of interest.

\section{References}

1. Tychynin, V.A.; Petrova, O.V. Nonlocal symmetries and formulae for generation of solutions for a class of diffusion-convection equations. J. Math. Anal. Appl. 2011, 382, 20-33.

2. Bluman, G.W.; Reid, G.J.; Kumei, S. New classes of symmetries for partial differential equations. J. Math. Phys. 1988, 29, 806-811.

3. Bluman, G.W.; Kumei, S. Symmetries and Differential Equations; Springer: New York, NY, USA, 1989.

4. Anderson, I.M.; Kamran, N.; Olver, P.J. Internal, external and generalized symmetries. Adv. Math. 1993, 100, 53-100.

5. Reyes, E.G. Nonlocal symmetries and the Kaup-Kupershmidt equation. J. Phys. A Math. Gen. 2005, 46, 073507:1-073507:19.

6. Bluman, G.W.; Doran-Wu, P. The use of factors to discover potential systems or linearizations. Acta Appl. Math. 1995, 41, 21-43.

7. Serov, M.; Omelyan, O.; Cherniha, R. Linearization of some systems of nonlinear diffusion equations by means of non-local transformations. Dop. Nat. Acad. Ukr. 2004, 10, 39-45.

8. Sophocleous, C. Potential symmetries of nonlinear diffusion-convection equations. J. Phys. A Math. Gen. 1996, 29, 6951-6959.

9. Lisle, I.G. Equivalence Transformations for Classes of Differential Equations. Ph.D. Thesis, University of British Columbia, Vancouver, BC, Canada, 1992.

10. Lisle I.G.; Reid G.J. Symmetry Classification Using Invariant Moving Frames; ORCCA Technical Report TR-00-08; University of Western Ontario: Ontario, Canada, 1992.

11. Popovych, R.O.; Ivanova, N.M. Potential equivalence transformations for nonlinear diffusion-Convection equations. J. Phys. A Math. Gen. 2005, 38, 3145-3155.

12. Anderson, R.L.; Ibragimov, N.H. Lie-B̈̈cklund Transformations in Applications; SIAM: Philadelphia, PA, USA, 1979.

13. Olver, P.J. Applications of Lie Groups to Differential Equations; Springer-Verlag: New York, NY, USA, 1993.

14. Bluman, G.W.; Cheviakov A.; Anco, S. Applications of Symmetry Methods to Partial Differential Equations; Applied Mathematical Sciences; Springer: New York, NY, USA, 2010; Volume 168, p. 367.

15. Bluman, G.W.; Yang, Z.Z. A symmetry-based method for constructing nonlocally related PDE systems. J. Math. Phys. 2013, 54, 093504:1-093504:22.

16. Fuschych, W.I.; Tychynin, V.A. Preprint No 82.33; Institut of Mathematics: Kiev, Ukraine, 1982.

17. Fuschych, W.I.; Tychynin, V.A. Exact solutions and superposition principle for nonlinear wave equation. Proc. Acad. Sci. Ukr. 1990, 5, 32-36. 
18. Tychynin, V.A. Nonlocal Symmetries and Solutions for Some Classes of Nonlinear Equations of Mathematical Physics. Ph.D. Thesis, Prydniprovsk State Academy of Civil Engineering and Architecture, Dnipropetrovsk, Ukraine, 1994.

19. Tychynin, V.A. Non-local symmetry and generating solutions for Harry-Dym type equations. J. Phys. A Math. Gen. 1994, 14, 2787-2797.

20. Tychynin, V.A.; Petrova, O.V.; Tertyshnyk, O.M. Symmetries and Generation of Solutions for Partial Differential Equations. SIGMA 2007, 3, 019:1-019:14.

21. Rzeszut, W.; Vladimirov, V.; Tertyshnyk, O.M.; Tychynin, V.A. Linearizability and nonlocal superposition for nonlinear transport equation with memory. Rep. Math. Phys. 2013, 72, 235-252.

22. Fushchych, W.I.; Serov, M.I.; Tychynin, V.A.; Amerov, T.K. On non-local symmetry of nonlinear heat equation. Proc. Acad. Sci. Ukr. 1992, 11, 27-33.

23. Storm, M.L. Heat conduction in simple metals. J. Appl. Phys. 1951, 22, 940-951.

24. Bluman, G.W.; Kumei, S. On the remarkable nonlinear diffusion equation $\frac{\partial}{\partial x}\left(a(u+b)-2 \frac{\partial u}{\partial x}\right)-\frac{\partial u}{\partial t}=0$. J. Math. Phys. 1980, 21, 1019-1023.

25. King, J.R. Some non-local transformations between nonlinear diffusion equations. J. Phys. A Math. Gen. 1990, 23, 5441-5464.

26. Cherniha, R.; Serov, M. Symmetries, Ansätze and Exact Solutions of Nonlinear Second-order Evolution Equations with Convection Terms, II. Eur. J. Appl. Math. 2006, 17, 597-605.

27. Rosen, G. Nonlinear heat conduction in solid. Phys. Rev. B 1979, 19, 2398-2399.

28. Cherniha, R.; Serov, M. Symmetries, Ansätze and Exact Solutions of Nonlinear Second-order Evolution Equations with Convection Terms. Eur. J. Appl. Math. 1998, 9, 527-542.

(c) 2015 by the author; licensee MDPI, Basel, Switzerland. This article is an open access article distributed under the terms and conditions of the Creative Commons Attribution license (http://creativecommons.org/licenses/by/4.0/). 